\title{
Experimental investigation on bubble coalescence under nonuniform temperature distribution in reduced gravity
}

\author{
Q. Kang *, H.L. Cui, L. Hu, L. Duan, W.R. Hu \\ National Microgravity Laboratory/CAS, Institute of Mechanics, Chinese Academy of Sciences, Beijing 100080, People's Republic of China
}

Received 20 September 2006; accepted 2 February 2007

Available online 13 February 2007

\begin{abstract}
Results on bubble coalescences from the space experiment of thermocapillary bubble migration conducted on board the Chinese 22nd recoverable satellite are presented in this paper. Some coalescences of large spherical bubbles under microgravity are observed through bubbles staying at the upper side of the test cell. The data of bubble coalescence time are recorded and compared with theoretical predictions, which is based on a theory to describe the tendency of coalescence connected to chemical potential difference. It is implied that the theory is applicable for the experimental data of bubble coalescence. Moreover, the angle between the line of two bubble centers and temperature gradient falled mostly in the range $20^{\circ}-40^{\circ}$.
\end{abstract}

(c) 2007 Elsevier Inc. All rights reserved.

Keywords: Bubble; Coalescence; Microgravity; Temperature gradient

\section{Introduction}

Bubble or drop coalescence in another immiscible liquid is a common physical phenomenon in material processing and welding on ground or in space. Many studies were carried out on the bubble and drop coalescence on ground, including measuring coalescence time (time that the bubble stays at the interface before coalescence occurs) [1-3]; changes in thickness of thin liquid film [4,5]; effects of applied force and approach velocity on coalescence [5]; effects of surfactants on coalescence $[1,3,6]$. Only a few studies related to the coalescence under microgravity $[1,7]$.

Coalescence time plays an important role in bubble or drop coalescence. Linton and Sutherland [8], Charles and Mason [9], Komasawa and Otake [10] discovered that the coalescence time increased with the increasing drop size when the drop coalesced with the plane of the homophase. Burrill and Woods [3] presented an equation describing the relation between coalescence time and drop size for the type of coalescence mentioned above. The equation is expressed as $t_{\text {coal }}=K_{n}(d)^{n^{\prime}}$, where $t_{\text {coal }}$

\footnotetext{
* Corresponding author.

E-mail address: kq@imech.ac.cn (Q. Kang).
}

is coalescence time, $d$ is the radius of the drop, $K_{n}$ and $n^{\prime}$ are the experimental fitting parameters. Based on a theory which describes the tendency of coalescence connected to chemical potential difference, $\mathrm{Pu}$ [11] proposed that coalescence time of two spherical drops varying with the drop radius can be expressed as

$\ln k_{d} \sigma\left(1 / r_{1}-1 / r_{2}\right)=k_{b} t_{\text {coal }}+b^{\prime}$,

where $r_{1}$ is the radius of small drop, $r_{2}$ is the radius of large drop, $\sigma$ is the interface tension, $t_{\text {coal }}$ is the coalescence time, $k_{d}$ is the thin film coefficient, $k_{b}$ and $b^{\prime}$ are the slope and the intercept of line fitting, respectively. In order to decrease the effect of approach velocity and drop deformation due to density difference between drop and immiscible liquid on coalescence, Chen and $\mathrm{Pu}$ [1] observed the mixed oil drops coalescence in water. The density of mixed oil is almost equal to the density of water. The experimental data were found agreeing with Eq. (1). But the method used by $\mathrm{Chen}$ and $\mathrm{Pu}$ can not prevent large size spherical bubbles from deforming on ground, therefore microgravity environment is necessary condition for studying large size spherical bubble coalescences.

Space experiment on thermocapillary bubble migration, bubble collision and bubble coalescence in a KF-96L series silicon oil of nominal viscosity 5 cst conducted on board the Chinese 


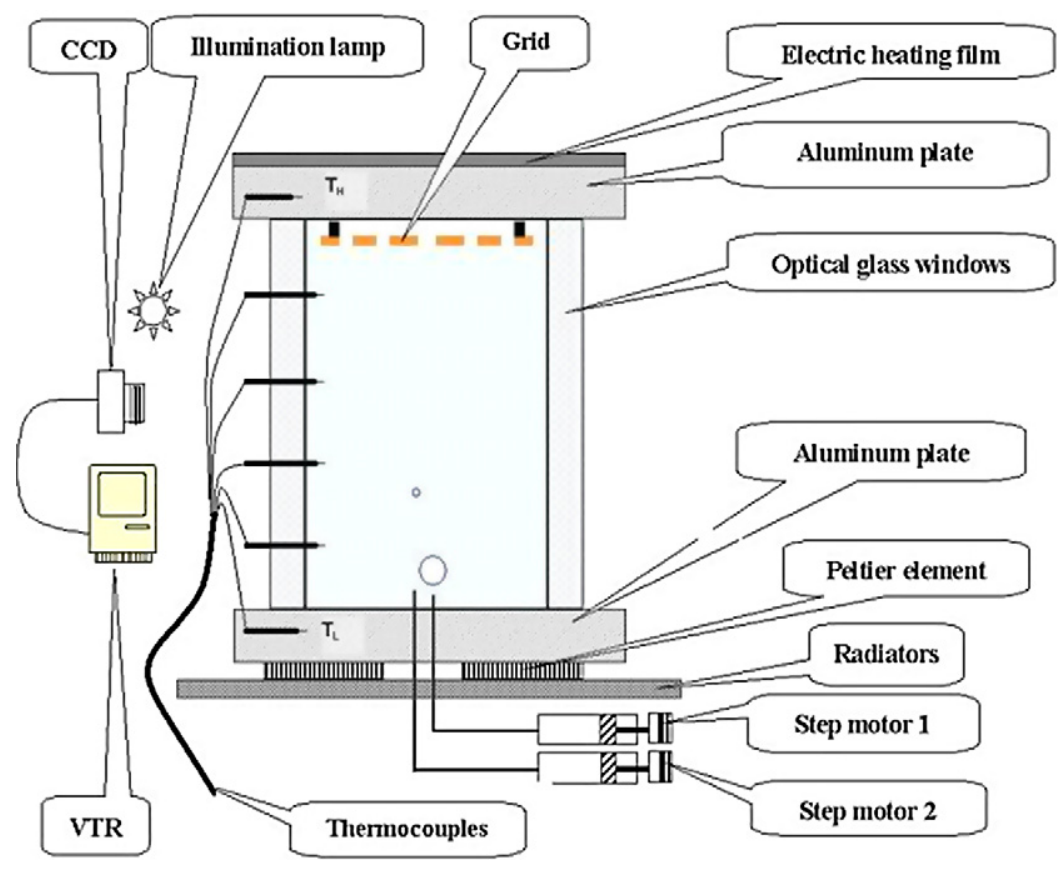

Fig. 1. Schematic diagram of experimental apparatus.

22nd recoverable satellite in 2005. Some bubble coalescences were observed through bubbles staying at the upper side of the cell. Experimental results on bubble coalescences are presented in this paper.

\section{Experimental apparatus}

As shown in Fig. 1, the cell is composed of four optical glass walls and two aluminum blocks at the top and the bottom, and the rectangular test cavity is $70 \mathrm{~mm}$ high with a horizontal cross-section of $40 \times 40 \mathrm{~mm}^{2}$. A piece of electric heating film and a Peltier element are placed at the top and the bottom surface, respectively, to maintain the temperature gradient. A grid plate is placed near the top to keep the uniformity of the temperature distribution and to prevent drops remaining at the top from disturbing temperature field. In order to ensure a steady applied temperature gradient, two proportional-integraldifferential temperature controllers are used to measure and maintain constant temperatures at the top and the bottom, respectively. Six thermocouples are used to measure the temperature field in the liquid. A cold light lamp composed of light emmitting diode (LED) was used to illuminate the cell in order to avoid the temperature disturbance from the light. There was an flexible vessel as a buffer connected with the top of the cell, which compensated for the increase in liquid volume with the increase of the temperature and collected the exhaust bubbles.

The whole process was controlled by the embedded system on board, which included controlling and measuring the temperature field, injection and detachment of bubbles, recording the experimental images. The heating process was started $100 \mathrm{~min}$ before bubble injection in order to obtain a linear and longitudinal temperature distribution. Each group bubbles were injected at an interval of several minutes. A system with a CCD camera and a videotape were used to record the bubble

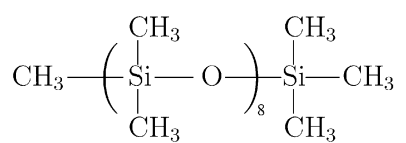

Fig. 2. Chemical formula of the KF-96L series silicon oil of nominal viscosity 5 cst.

Table 1

Physical property parameters of $5 \mathrm{cSt}$ silicon oil

\begin{tabular}{llllllll}
\hline Temperature $\left({ }^{\circ} \mathrm{C}\right)$ & 15.0 & 25.0 & 35.0 & 50.0 & 57.0 & 70.0 & 75.0 \\
Density $\rho\left(\mathrm{kg} / \mathrm{m}^{3}\right)$ & 919 & 918.5 & 902 & 887 & 882.3 & 869 & 864.2 \\
Thermal conductivity & 0.1121 & 0.111 & 0.1099 & 0.1083 & 0.1076 & 0.1054 & 0.1045 \\
$\lambda(\mathrm{W} / \mathrm{m} \mathrm{K})$ & & & & & & & \\
$\begin{array}{l}\text { Dynamic viscosity } \\
\mu\left(10^{-3} \times \mathrm{N} \mathrm{s} / \mathrm{m}^{2}\right)\end{array}$ & 5.09 & 4.27 & 3.61 & 2.86 & 2.67 & 2.17 & 2.01 \\
$\begin{array}{l}\text { Surface tension } \\
\sigma\left(10^{-5} \times \mathrm{N} / \mathrm{m}\right)\end{array}$ & $2101-7.8 \times$ temperature & & & \\
\hline
\end{tabular}

tracks which would be analyzed after flight. The output format of video is PAL and the video rate was 25 frames per second. The time resolution was $0.04 \mathrm{~s}$, and the image resolution was $0.1 \mathrm{~mm}$ per pixel.

A KF-96L series silicon oil of nominal viscosity 5cst made in Shin-Etsu Chemical Co., Ltd. of Japan was selected as the continuous phase. The silicon oil as a kind of dimethyl silicon oil is an ideal experimental material about surface phenomenon, because of its steady surface tension for contamination with many materials. The oil could not be dissolved by water or alcohol. The chemical formula of the oil is shown in Fig. 2 and the molecule weight is 680 . The surface tension was measured by a plate method using a kruss k12 tensiometer. Surface tension and other physical property parameters of the 5cSt silicon oil coming from Xie [12] are listed in Table 1. Air was used as the dispersed phase. The experimental cell had been cleaned 


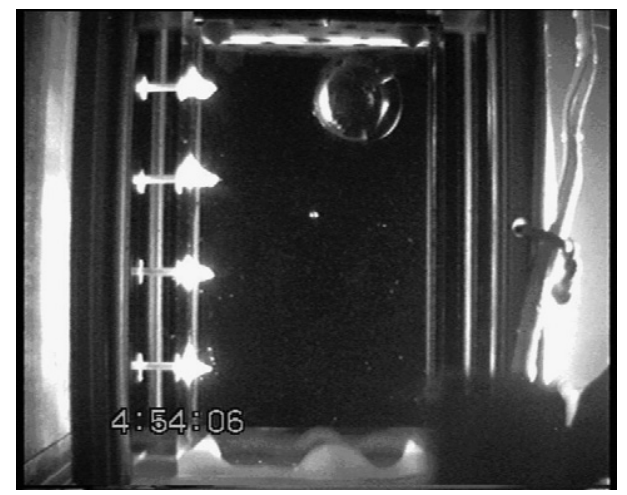

Fig. 3. Photo of a bubble staying under the grid.

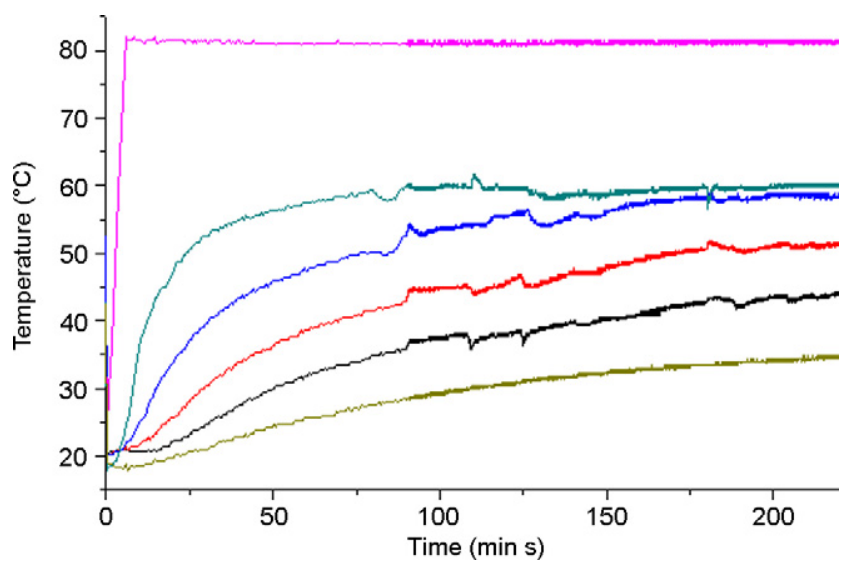

Fig. 4. Temperature measured with thermocouples.

carefully before filled with the silicon oil without any surfactant, therefore the influence by surfactants on the properties of surface was neglected.

\section{Experimental results and analysis}

The peak acceleration level aboard the satellite during the experiment was less than $10^{-3} g_{0}$ from the monitored data, where $g_{0}$ is the acceleration of gravity at the surface of the earth, therefore the influence from the buoyancy force could be ignored.

In the experiment, some bubbles stayed under the grid shown in Fig. 1. Fig. 3 shows a bubble whose radius is $7 \mathrm{~mm}$ at the 150th minute. The total space experiment lasted $220 \mathrm{~min}$. The heating and cooling process started $100 \mathrm{~min}$ before bubble injection. Temperatures measured with thermocouples are shown in Fig. 4, where six curves from the top down correspond to the six thermocouples from the top down, respectively. It is shown in Fig. 4 that the temperature field was disturbed by the accumulated upper bubbles.

Bubble coalescences were observed through bubbles staying at the upper side of the cell. The process of two-bubble touch and coalescence is shown in Fig. 5. The radius of bubbles are 6.69 and $4.99 \mathrm{~mm}$. The smaller touched the larger at the position as shown in Fig. 5a; then the smaller moved to the position below the larger along the interface of the larger as shown in Fig. 5b, and the coalescence angle $\theta$ (the angle between the line
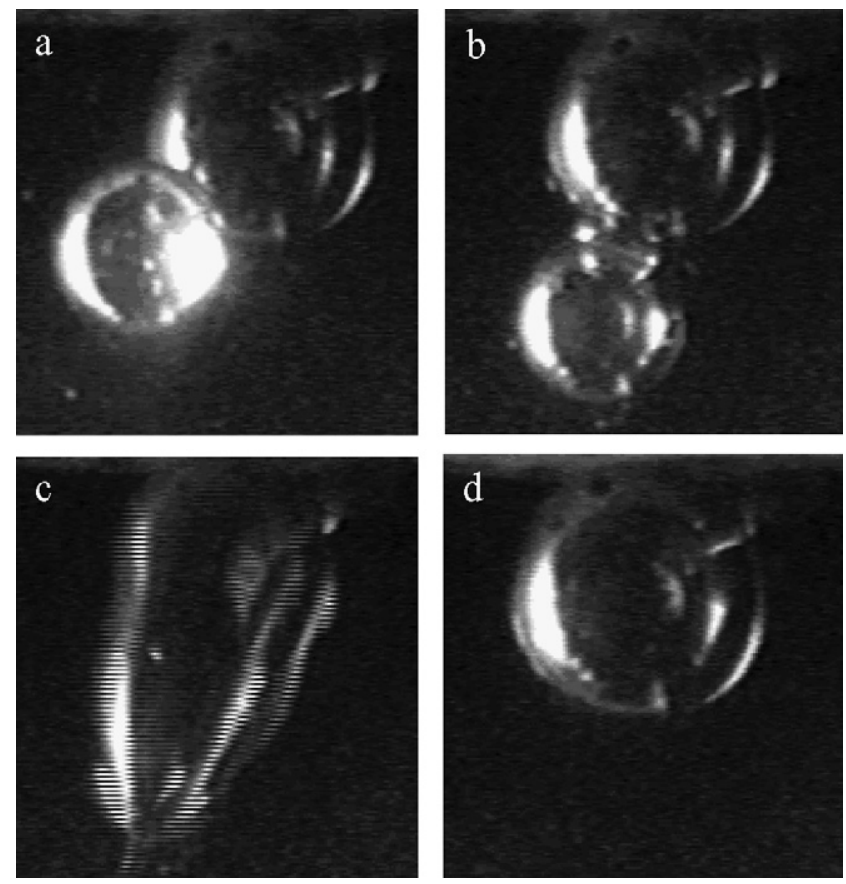

Fig. 5. Process of bubble coalescence, (a) initial position of touch, (b) position of coalescence, (c) instant of coalescence happening, and (d) new bubble after coalescence.

of two-bubble centers and initial undisturbed temperature gradient) was $18.2^{\circ}$; lastly bubble coalescence happened as shown in Fig. 5c, and new bubble was shown in Fig. 5d. The coalescence time from touch to coalescence was $27 \mathrm{~s}$.

The value of the temperature gradient around the bubble could be estimated from the second and the third curves in Fig. 4 from the top down, because all the coalescence happened at the upper side of the cell. During the process of the bubble coalescence, the temperature gradient was not more than $0.2^{\circ} \mathrm{C} / \mathrm{mm}$, which resulted in a weak thermocapillary flow around the two bubbles. Moreover the speed of the moving bubble was very small $(<0.5 \mathrm{~mm} / \mathrm{s})$ observing from the experimental video. The dimensionless number We $<10^{-6}$ shown as Eq. (2) could describe the ratio of inertia force to surface tension. Such small We means that the influence of the momentum on the surface phenomenon could be negligible.

$\mathrm{We}=\frac{\rho_{\mathrm{b}} V^{2} R_{\mathrm{b}}}{\sigma}$,

where $\rho_{\mathrm{b}}, V$, and $R_{\mathrm{b}}$ are the density, the velocity, and the radius of the air bubble, respectively.

The data of bubble size and the coalescence time listed in Table 2. The relationship between $\ln \left(1 / r_{1}-1 / r_{2}\right)$ and $t_{\text {coal }}$ is shown in Fig. 6, and the line fitting is expressed as

$$
\begin{aligned}
\ln \left(1 / r_{1}-1 / r_{2}\right)= & (-0.77 \pm 0.03) \\
& +(-0.077 \pm 0.002) \times t_{\text {coal }} .
\end{aligned}
$$

As shown in Fig. 6, large size bubbles kept sphere and coalescence time was consistent with Eq. (1), although there was weak thermocapillary flow. It is inferred that the theory which describes the tendency of coalescence connected to chemical 
Table 2

Size of bubbles, coalescenece time, and coalescence angle

\begin{tabular}{rlllrl}
\hline No. & $\begin{array}{l}r_{2} \\
(\mathrm{~mm})\end{array}$ & $\begin{array}{l}r_{1} \\
(\mathrm{~mm})\end{array}$ & $\ln \left(1 / r_{1}-1 / r_{2}\right)$ & $\begin{array}{l}t_{\text {coal }} \\
(\mathrm{s})\end{array}$ & $\begin{array}{l}\text { Coalescence } \\
\text { angle }\left(^{\circ}\right)\end{array}$ \\
\hline 1 & 3.1 & 2.1 & -1.9 & 11.04 & 56 \\
2 & 2.1 & 1.1 & -0.8 & 3.20 & 54 \\
3 & 3.5 & 1.0 & -0.3 & 1.20 & 15 \\
4 & 4.9 & 3.4 & -2.4 & 23.00 & 39 \\
5 & 5.2 & 4.2 & -3.1 & 23.00 & 16 \\
6 & 6.0 & 3.9 & -2.4 & 17.64 & 9 \\
7 & 2.2 & 1.1 & -0.8 & 5.00 & 70 \\
8 & 6.4 & 3.4 & -2.0 & 12.00 & 29 \\
9 & 6.7 & 5.0 & -3.0 & 27.00 & 18 \\
10 & 7.5 & 3.9 & -2.1 & 15.12 & 36 \\
11 & 2.0 & 1.1 & -0.9 & 6.04 & 36 \\
12 & 2.0 & 1.2 & -1.1 & 1.20 & 31 \\
13 & 7.6 & 2.6 & -1.4 & 9.24 & 21 \\
14 & 7.8 & 2.6 & -1.4 & 9.48 & 20 \\
15 & 5.0 & 1.6 & -0.9 & 1.72 & 56 \\
16 & 8.2 & 5.0 & -2.6 & 28.00 & 39 \\
17 & 8.9 & 1.6 & -0.7 & 3.60 & 31 \\
18 & 8.9 & 3.9 & -1.9 & 19.68 & 30 \\
19 & 9.3 & 3.0 & -1.5 & 10.32 & 39 \\
\hline
\end{tabular}

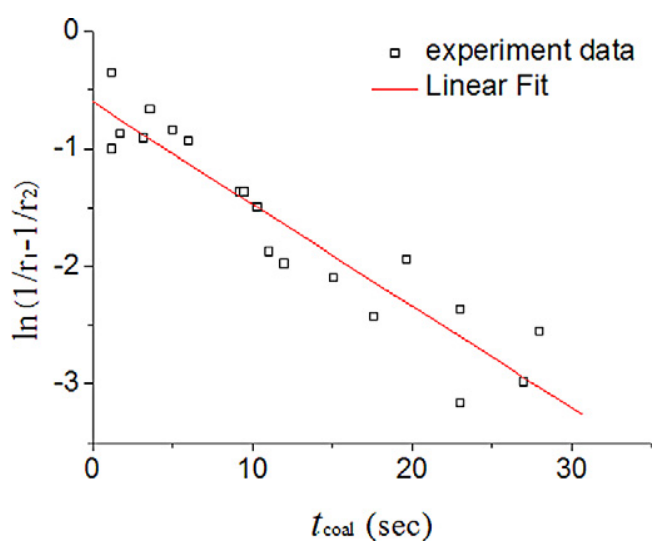

Fig. 6. Relationship between $\ln \left(1 / r_{1}-1 / r_{2}\right)$ and $t_{\text {coal }}$.

potential difference and Eq. (1) are reasonable. When the temperature gradient is small, the effect of the weaker thermocapillary flow on bubble coalescence time is not significant.

Now we called that the approaching bubble is bubble 1 , and the rest bubble is bubble 2. After touching bubble 2, bubble 1 glided along the interface of bubble 2 . The distribution of the coalescence angle $\theta$ is shown in Fig. 7. The probability of the angle ranging from $30^{\circ}$ to $40^{\circ}$ was largest, and the probability of ranging from $20^{\circ}$ to $40^{\circ}$ was $57.9 \%$. It is possible that the flow induced by temperature gradient resulted in the feature of the coalescence angle as shown in Fig. 7. The effect of thermocapillary flow on the coalescence angle needs further theoretical and numerical research.

\section{Conclusion}

The experimental data on bubble coalescences from the space experiment of thermocapillary bubble migration con-

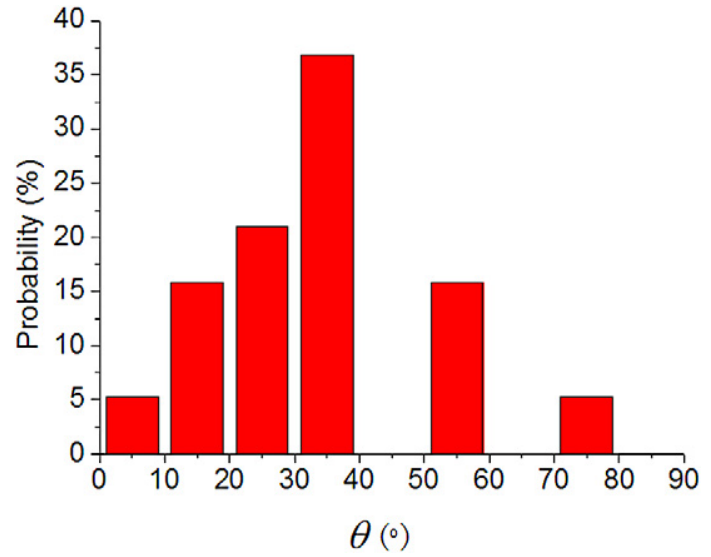

Fig. 7. Probability of coalescence angle $\theta$.

ducted on board the Chinese 22nd recoverable satellite are reported. Bubble coalescences are observed through bubbles staying at the upper side of the test cell. A total of 19 coalescences are recorded, and coalescence time and size of bubbles are fitted with a formula derived from the theory that the coalescence phenomenon is driven by chemical potential difference between bubbles or drops. However the thermocapillary force made the bubble glide and affected the position of bubble coalescence under nonuniform temperature distribution in the experiment. And the angle between the line of two bubble centers and temperature gradient falled mostly in the range $20^{\circ}-40^{\circ}$.

\section{Acknowledgments}

This work was supported by the National Nature Science Foundation of China (10432060) and Knowledge Innovation Program of Chinese Academy of Sciences (KJCX2-SW-L05, KSCX2-SW-322).

\section{References}

[1] D.H. Chen, B.Y. Pu, J. Colloid Interface Sci. 243 (2001) 433.

[2] T. Gillespie, E.K. Rideal, Trans. Faraday Soc. 52 (1956) 173.

[3] K.A. Burrill, D.R. Woods, J. Colloid Interface Sci. 42 (1973) 35.

[4] S.A.K. Jeelani, S. Hartland, Chem. Eng. Sci. 46 (1991) 1807.

[5] G.F. Scheele, D.E. Leng, Chem. Eng. Sci. 26 (1971) 1867.

[6] G.W. Stevens, H.R.C. Pratt, D.R. Tai, J. Colloid Interface Sci. 136 (1990) 470.

[7] P. Guenoun, Y. Jayalakshmi, Suprieure, B. Khalil, D. Beysens, Y. Garrabos, B. Le Neindre, Phase Separation in Fluids without Gravity: Hydromechanics and Heat/Transfer in Microgravity, Gordon \& Breach, New York, 1991, p. 279.

[8] M. Linton, K.L. Sutherland, J. Colloid Interface Sci. 11 (1956) 391.

[9] G.E. Charles, S.G. Mason, J. Colloid Interface Sci. 15 (1960) 236.

[10] I. Komasawa, T. Otake, J. Chem. Eng. Jpn. 3 (1970) 243.

[11] B.Y. Pu, Ph.D. dissertation, Swiss Fed. Inst. Technol., Zurich, 1995.

[12] J.C. Xie, H. Lin, P. Zhang, F. Liu, W.R. Hu, J. Colloid Interface Sci. 285 (2005) 737. 